\title{
Audit on Incidences of Oxaliplatin Induced Hypersensitivity Reactions during Infusion in Day Care Oncology at a Tertiary Care Hospital, Karachi, Pakistan
}

\author{
Arifa Aziz ${ }^{1 *}$, Muhammad Nawaz Khan ${ }^{2}$ and Shafaq Zubair ${ }^{2}$ \\ 1Staff Medical Officer/Supervisor Day care Oncology, Department of Oncology, The Aga Khan University Hospital, Karachi, Pakistan \\ ${ }^{2}$ Medical Officer, Department of Oncology, The Aga Khan University Hospital, Karachi, Pakistan
}

${ }^{\star}$ Corresponding author: Dr. Arifa Aziz, Staff Medical Officer/Supervisor Day care Oncology, Department of Oncology, The Aga Khan University Hospital, Karachi, Pakistan; Cell: +92323-2517677; Email: arifa.aziz@aku.edu

Received: February 10, 2022; Accepted: February 17, 2022; Published: February 22, 2022

\begin{abstract}
Objective: Due to increased occurrence of hypersensitivity reactions with Oxaliplatin in the preceding months, it was decided to conduct an audit on incidences of hypersensitivity reactions induced by Oxaliplatin during infusion and measures that can be taken to prevent such reactions.

Method: We performed a prospective observational study of patients who admitted for oxaliplatin based chemotherapy between $16^{\text {th }}$ October 2019 and $26^{\text {th }}$ December 2019.

All patients coming to oncology day care for infusion of oxaliplatin based chemotherapy protocols were included in this audit.

Inclusion Criteria: (1) Patients aged more than 18 years age of both genders. (2) Patients coming for chemotherapy regimen with oxaliplatin.

Exclusion Criteria: (1) Patients with known hypersensitivity to other chemotherapeutic agents in an Oxaliplatin based regimen. (2) All patients receiving oxaliplatin anywhere except daycare oncology facility.

Result: OXALIPLTIN AUDIT RESULTS • Total number of patients: $109 \bullet$ No Reaction: $86 \bullet$ Reaction without prior intervention: $6 \bullet$ No reaction after prior intervention: $9 \bullet$ Reaction with prior intervention: 8 .

Conclusion: The number of patients with hypersensitivity reactions is considerably high, therefore we decided to give injection solumedrol, antihistamine and prolong the duration of infusion in patients who experienced hypersensitivity reactions in previous cycles and these premedication measures significantly declined the incidents of hypersensitivity reactions due to Oxaliplatin.
\end{abstract}

Keywords: Drug reaction, Oxaliplatin, Prolong duration of infusion, Steroids, Antihistamine

\section{Introduction}

Oxaliplatin is a chemotherapeutic drug mostly used in gastrointestinal malignancies mostly in colorectal cancers with excellent results but it was noticed during last few months that oxaliplatin induced hypersensitive reactions increasing with time therefore we decided to do audit in order to find out the number of incidents, reason and ways to manage these types of reactions which usually presented during infusions, common side effects noticed were choking feeling, difficulty in swallowing, numbness of legs and difficulty in walking these symptoms settle within 60 minutes, roughly between 30 minutes to 60 minutes and these side effects were very distressing for patients.

\section{Methods}

This prospective observational study is designed to analyze and measure the incidences of hypersensitivity reactions to patients receiving Oxaliplatin infusions. Their initial assessment was performed which includes the height, weight, sex, diagnosis, and cycles per protocol identified. Data of patients was collected from 16th October 2019 till 26th December 2019. All patients above 18 years of age, from both genders, coming to oncology day care for infusion of Oxaliplatin based chemotherapy protocols were included in this audit. The Exclusion criteria excluded all the patients who were receiving Oxaliplatin based chemotherapy regimens anywhere except the daycare facility and all those who had a history of known hypersensitivity to other chemotherapeutic agents in an Oxaliplatin based regimen.

The Medical Record numbers were noted alongside of the patient's name, type of reaction and the relevant treatment management collected. The Standard time of infusion of oxaliplatin as per international protocol was set at 2 hours. In the patients with a history of hypersensitivity reaction oxaliplatin infusion time was prolonged along with administration of intravenous Hydrocortisone and intravenous Pheniramine maleate. The incidence and findings of 
hypersensitivity reactions were then recorded on the same day as well as on the following cycles. Those with a known history of hypersensitivity reaction were given either intravenous Hydrocortisone/ Pheniramine maleate or prolonged Infusion time or a combination of these. The patients were afterwards observed for the signs and symptoms of hypersensitivity if any and the findings were then recorded. The study was performed to observe the incidence of hypersensitivity reactions in patients receiving oxaliplatin based regimens in a daycare setting. Those with a known history of hypersensitivity reaction were given either injection hydrocortisone or injection Pheniramine maleate or duration of infusion is Prolonged, but in few patients all three interventions were done.

\section{Inclusion Criteria}

(1) Patients age more than 18 years of both gender.

(2) Patients coming for chemotherapy regimen with oxaliplatin at day care oncology.

\section{Exclusion Criteria}

(1) Patients less than 18 years of age.

(2) Reactions with other chemotherapeutic drugs in regimen will not be considered.

\section{Results}

A total of a hundred and nine sessions of oxaliplatin infusion were recorded eighty-six of these were uneventful the remaining twentythree sessions with oxaliplatin were recorded with hypersensitivity reactions out of these six sessions were without prior history of hypersensitivity reaction while the rest 17 comprised of the sessions of patients with a known history of hypersensitivity to Oxaliplatin. In 9 out of the 17 sessions the patients received intravenous hydrocortisone or intravenous Pheniramine maleate or a combination of both without prolonging the infusion time above the standard of 2 hours. In the remaining 8 patients the infusion time was also prolonged to a maximum of 4 hours along with receiving intravenous hydrocortisone and intravenous Pheniramine maleate while the rest had documented hypersensitivity reactions as shown in Figure 1.

A total of 109 Oxaliplatin based regimens infusion sessions were observed,86 of these sessions was uneventful without any reaction or history of previous cycles hypersensitivity reaction. The remaining 23 sessions included 6 sessions without any prior history of hypersensitivity to oxaliplatin while the other 17 comprised of the sessions of patients with a known history of hypersensitivity to Oxaliplatin in particular. In 9 out of the 17 sessions the patients received intravenous hydrocortisone or intravenous Pheniramine maleate or a combination of both without prolonging the infusion time above the standard of 2 hours. In the remaining 8 patients the infusion time was also prolonged to a maximum of 4 hours along with receiving intravenous hydrocortisone and intravenous Pheniramine maleate 9 out of 17 sessions in patients with known hypersensitivity had uneventful infusions after receiving interventions like injection Hydrocortisone, Pheniramine maleate (as pre-chemotherapy medications added to the standard pre-chemotherapy regimen) and duration of infusion is also prolonged. The rest 8 showed symptoms of mild to moderate hypersensitivity reactions to Oxaliplatin such as rash, itching, hoarseness of voice even after receiving the interventions with intravenous injections of hydrocortisone and pheniramine maleate time duration of infusion was 2 hours. No patient complained of difficulty in breathing and none of the patients in these 23 sessions developed anaphylaxis to Oxaliplatin. All the patients had their complaints resolved within a maximum of 60 minutes and therefore were discharged home in a stable condition.

Administration of injections Pheniramine maleate, hydrocortisone along with prolonged infusion time, in nine out of the seventeen sessions of chemotherapy had uneventful outcome. The rest eight patients' infusions reported mild to moderate reactions which were managed smoothly with intravenous hydrocortisone, intravenous Pheniramine maleate at day care oncology.

\section{OXALIPLTIN AUDIT RESULTS}

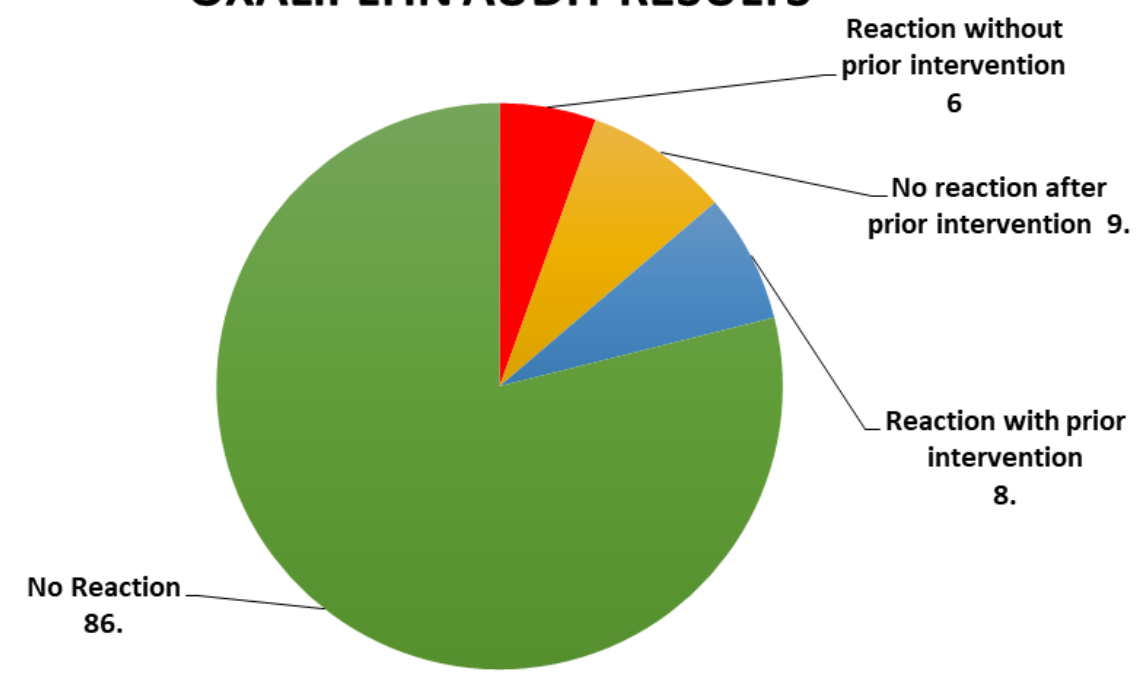

Figure 1: OXALIPLTIN AUDIT RESULTS • Total number of patients: $109 \bullet$ No Reaction: $86 \bullet$ Reaction.23-Reaction without prior intervention: $6 \bullet$ No reaction after prior intervention: 9 . Reaction with prior intervention: 8 . 


\section{Conclusion}

While the incidence of hypersensitivity to oxaliplatin is common, adding intravenous injection hydrocortisone and intravenous injection Pheniramine maleate to the standard pre-chemotherapy regimens and prolonging infusion times in patients with known hypersensitivity, can considerably reduce the severity and progression of these symptoms in subsequent cycles. Furthermore, the chances of regimen change solely due to Oxaliplatin hypersensitivity are also reduced to negligible following these interventions.

\section{Discussion}

Oxaliplatin is a very commonly used chemotherapeutic drug in gastrointestinal malignancies Oxaliplatin is placed in third generation platinum compound and is the most effective first line chemotherapeutic agent for colorectal cancer in combination with $5 \mathrm{FU}$ and leucovorin. It is indicated for pancreatic, gastric, and testicular cancers combined with bevacizumab, capecitabine, irinotecan and other cytotoxic agents. However, moderate to severe hypersensitivity reactions during or after oxaliplatin infusion usually require cessation of chemotherapy or substitution of the key therapeutic drug oxaliplatin with some other less effective chemotherapeutic drug which largely interferes with improved patient prognosis [1]. In patients who even after receiving premedication with intravenous hydrocortisone and intravenous Pheniramine maleate need to start with desensitization protocol which in such cases are very effective $[2,3]$.

Oxaliplatin is a very commonly used chemotherapeutic drug in gastrointestinal malignancies, it was noticed that during infusion patients' complaint of numbness of hands and very occasionally complaint of numbness of legs with difficulty in walking which lasts for few minutes, rash all over the body and itching, difficulty in swallowing and choking feelings which last for few minutes. Hypersensitivity reactions facial flushing, tongue swelling, pruritic, tachycardia, dyspnea, headache, chills, fever, burning sensation and dizziness mostly seen with oxaliplatin in colorectal cancer. Fever alone is not the only symptom of oxaliplatin hypersensitivity reaction, but this may indicate that patient may develop serious reactions in following cycles as seen international study of papers published which show patients' blood pressure was $95 / 43 \mathrm{~mm}$, pulse 120 /min and oxygen saturation $88 \%$ to $90 \%$ patient was hospitalized for management and recovered in 8 hours [4-5].

In few cases serious hypersensitivity reactions were reported in $12 \%$ of patients treated with oxaliplatin, whereas $1 \%$ of these patients may face a life-threatening situation. Extended steroid premedication with slower oxaliplatin infusion rate can be employed for safety in patients after severe hypersensitivity reaction with oxaliplatin, intravenous dexamethasone or hydrocortisone in prechemotherapy was added with oxaliplatin and will be considered very effective in decreasing the hypersensitivity reactions [6].

Total number of entries of patients were hundred and nine. Patients without reaction 86, Patients with reaction 23. The remaining 23 sessions included 6 sessions without any prior history of hypersensitivity to oxaliplatin while the other 17 comprised of the sessions of patients with a known history of hypersensitivity to Oxaliplatin, 9 out of the 17 sessions the patients received intravenous injection hydrocortisone and intravenous injection Pheniramine maleate as prechemotherapy and finish infusion in 4 hours and 8 numbers of patients received intravenous injection hydrocortisone and intravenous injection Pheniramine maleate without increase duration of infusion and finish in 2-hours, number of patients not given injection anti-histamines and injection intravenous hydrocortisone 0 , in 17 number of patients who had reaction for the first time 6, number of patients who had reaction in follow up cycles 17.

Treatment and prevention consist of increase duration of infusion plus intravenous antihistamine, and use of intravenous steroids $[7,8]$.

\section{Way Forward}

Desensitization protocol could help patients who experience severe hypersensitivity reactions and patient can receive treatment with oxaliplatin which consider as active and effective treatment for colorectal cancer.

\section{References}

1. Bano N, Najam R, Mateen A (2013a) Neurological adverse effects in patients of advanced colorectal carcinoma treated with different schedules of FOLFOX. Chemother Res Pact.

2. Arotcarena R, Barthelemy P, Piot M, et al. (2001) Read ministration of oxaliplatin using a rapid desensitization method after severe anaphylactic reaction. Gastroenterol Clin Biol 25: 206-7.

3. Alvarez-Cuesta E, Madrigal-Burgaleta R, Angel-Pereira D, et al. (2015) Delving into cornerstones of hypersensitivity to antineoplastic and biological agents: value of diagnostic tools prior to desensitization. Allergy 70: 784-794. [crossref]

4. Anderson BJ, Peterson LL (2015) Systemic capillary leak syndrome in a patient receiving adjuvant oxaliplatin for locally advanced colon cancer. J Oncol Pharm Pract. [crossref]

5. Alliot C, Messouak D, Beets C, et al. (2001) Severe anaphylactic reaction to oxaliplatin. Clin Oncol (R Coll Radiol) 13: 236.

6. Benedik J (2015) Hypersensitivity: case report. Reactions.137. Bahl M, Dean T (2015) An intermediate step for the management of hypersensitivity to platinum and taxane chemotherapy. Curr Oncol 22: 220.

7. Brockow K, Przybilla B, Aberer W, et al. (2015) Guideline for the diagnosis of drug hypersensitivity reactions. Allergo J Int 24: 94-105. [crossref]

8. Bano N, Najam R, Qazi F, et al. (2014) Gastrointestinal Adverse Effects in Advanced Colorectal Carcinoma Patients Treated with Different Schedules of FOLFOX. Asian Pac J Cancer Prev 15: 8089-93. [crossref]

\section{Citation:}

Aziz A, Nawaz Khan M, Zubair S (2022) Audit on Incidences of Oxaliplatin Induced Hypersensitivity Reactions during Infusion in Day Care Oncology at a Tertiary Care Hospital, Karachi, Pakistan. Cancer Stud Ther J Volume 7(2): 1-3. 